\title{
Carga horária de cirurgia em escolas médicas do Brasil
}

\author{
Surgery course load in Brazilian medical schools
}

\author{
Athos Paulo Santos Martini' (D) athosmartini@gmail.com \\ Sofia Romay Oliveira' (D) sromay10@gmail.com \\ Suely Grosseman ${ }^{1,2}$ (D) sgrosseman@gmail.com
}

\section{RESUMO}

Introdução: A cirurgia é um importante componente dos currículos dos cursos de graduação em Medicina. Este estudo foi realizado por não se conhecer a carga horária de cirurgia nas escolas médicas brasileiras.

Objetivo: Analisar a carga horária de cirurgia de escolas médicas brasileiras.

Método: Este estudo foi transversal e descritivo, realizado em escolas médicas brasileiras reconhecidas pelo Ministério da Educação que iniciaram atividades até 31 de dezembro de 2017 e disponibilizavam matriz curricular e/ou projeto político-pedagógico com carga horária de cirurgia na internet até setembro de 2018. As variáveis estudadas foram carga horária total e de cirurgia antes do internato e durante esse período, região geográfica e gratuidade das escolas. Os dados foram analisados por meio de estatística descritiva, teste t de Student, análise de variância e testes U de Mann-Whitney e de Kruskal-Wallis, rejeitando-se a hipótese nula quando $p<0,05$.

Resultados: Foram incluídas 205 das 323 escolas médicas existentes, das quais 175 disponibilizavam carga horária de cirurgia no internato; 157, antes do internato; e 129, disponibilizavam ambas as cargas horárias. A mediana da carga horária total de cirurgia foi de 815,0 horas $\left(P_{25-75}=677,5-992,0\right.$; limites: $340,0-1.665,0$ horas), e a carga horária média antes do internato foi de 268,7 horas (desvio padrão $=140,3$; limites: $32,0-780,0)$, ambas sem diferença estatística por região geográfica ou gratuidade da escola. A mediana da carga horária do internato foi de 540,0 horas $\left(P_{25-75}=400,0\right.$ - 712,0; limites: 170,0 - 1.410,0), sem diferença estatística por região geográfica, mas maior nas escolas gratuitas. Em relação à carga horária total do currículo do curso, a porcentagem média da carga horária de cirurgia antes do internato foi $3,2 \%$ (desvio padrão $=1,7)$; a porcentagem mediana da carga horária de cirurgia no internato, 6,4\% $\left(P_{25-75}=5,0-\right.$ 8,2); e a porcentagem mediana da carga horária de ambos os períodos foi de, $9,7 \%\left(P_{25-75}=8,3-11,8\right)$.

Conclusões: Apesar da variação nos limites extremos da carga horária de cirurgia, a mediana de seu total e sua média antes do internato são semelhantes por região geográfica e gratuidade da escola, e sua mediana no internato é também semelhante por região, mas maior em escolas médicas gratuitas. Os valores encontrados neste estudo podem ajudar os gestores na avaliação e no planejamento da carga horária de cirurgia em suas escolas.

Palavras-chave: Educação Médica; Cirurgia, Currículo; Carga Horária.

\section{ABSTRACT}

Introduction: Surgery is an important curricular component of undergraduate medical courses. This study was conducted because the surgery course load in Brazilian medical has not been systematically explored at the national level.

Objective: To analyze the surgery course load in Brazilian medical schools.

Method: A cross-sectional, descriptive study was carried out with Brazilian medical schools acknowledged by the Ministry of Education, which had begun their activities prior to December 31, 2017 and, as of September 2018, had their curriculum matrix and/or political-pedagogical project with the surgery course load available on the internet. The variables studied were total medical curriculum course load and surgery course load before and during clerkship, as well as the schools' geographic region and fee status. Data analysis was performed using descriptive statistic Student $t$-test, analysis of variance, and Mann-Whitney $U$ and Kruskal-Wallis tests, with the null hypothesis rejected for $p<.05$.

Results: The study included 205 of the country's 323 existing medical schools, of which 175 had available information on the surgery course load during the clerkship, 157 before the clerkship, and 129 had information on course load before and after the clerkship. The median total surgery course load in hours was $815.0\left(P_{25.75}=677.5-992.0 ;\right.$ minimum $=340.0 \mathrm{~h} ;$ maximum $\left.=1,665.0\right)$, while the mean surgery course load before clerkship in hours was $268.7(S D=140.3 ;$ minimum $=32.0$; maximum $=780.0)$, with no difference between geographic regions or fee status. During the clerkship, the median course load was 540.0 hours $\left(P_{25.75}=400.0-712.0\right.$; minimum $=170.0$ h; maximum $\left.=1,410.0\right)$, with no difference between geographic regions, but with higher values in medical schools with no tuition fees. Regarding the total curriculum course load, the mean percentage of the surgery course load before clerkship was 3.2\% $(S D=1.7)$, the median percentage during the clerkship was $6.4 \%\left(P_{25-75}=5.0-8.2\right)$, the median percentage of the total surgery course load was $6.4 \%\left(P_{25-75}=5.0-8.2 \%\right.$, and the median percentage of surgery course load (both periods) was $9.7 \%\left(P_{25-75}=8.3-11.8 \%\right)$.

Conclusions: Despite the considerable variation in the surgery course load limits, the median of total surgery and the mean of surgery course load before clerkship were similar across geographic regions and fee statuses. The median surgery course load during clerkship was also similar across regions but higher in tuition-free medical schools. The values found in this study can help schools' administrators to assess and plan the surgery course load in their institutions.

Key words: Medical Education; Surgery; Curriculum; Course load.

1 Universidade Federal de Santa Catarina, Florianópolis, Santa Catarina, Brasil.

2 Faculdade Pequeno Príncipe, Curitiba, Paraná, Brasil.

Editora-chefe: Daniela Chiesa $\quad$ Editora associada: Daniela Chiesa

Recebido em 19/06/20; Aceito em 27/01/21. Avaliado pelo processo de double blind review. 


\section{INTRODUÇÃO}

A cirurgia é uma das grandes áreas da medicina, sendo um componente do currículo de graduação em Medicina. No passado, esperava-se que um médico recém-formado fosse capaz de realizar cirurgias. Porém, atualmente, há a expectativa de que o tempo destinado ao aprendizado sobre detalhes das técnicas cirúrgicas das especialidades não ocupe muito espaço do currículo, deixando esse ensino para a residência médica em cirurgia ${ }^{1-4}$.

Muitos fatores contribuíram para essa mudança na educação médica e em todas as áreas do saber. Na década de 1970, Bohoslavsky já apontava características inadequadas no modelo existente da educação universitária na maior parte do mundo. Havia uma discrepância entre a organização educacional e a estrutura produtiva que gerava uma formação de profissionais cuja prática não era alinhada às necessidades do país. Havia também a segmentação do saber em fragmentos, pela dificuldade ideológica de ver a realidade de forma mais integrada, em sua complexidade, o que resultava na formação de especialistas em fragmentos da realidade. Como consequência, havia uma importante cisão entre saber e fazer, cognição e ação, e universidade e realidade ${ }^{5}$.

Na educação médica, essa fragmentação exacerbou-se com a reforma do currículo proposta por Flexner em 1910', adotada por uma maioria de escolas médicas (EM), e, em paralelo, com o aumento acelerado e contínuo na produção de conhecimentos, acarretando aumento progressivo na carga horária $(\mathrm{CH})$ dos currículos de Medicina e sobrecarga teórica para os estudantes ${ }^{7,8}$.

Diante dessa realidade, em todo o mundo, foram organizados movimentos visando ao alinhamento da formação médica às necessidades de aprendizagem dos estudantes e às necessidades de saúde individuais e coletivas da população. Esse processo intensificou-se a partir das décadas de 1970 e $1980^{9}$, e, a partir dos anos 1990, foram elaborados projetos, recomendações e diretrizes para os cursos de Medicina, entre eles Tomorrow's doctors, no Reino Unido ${ }^{10,11}$, CanMEDS, no Canadá12,13, Outcome-based education ${ }^{14}$ e Processo de Bologna ${ }^{15}$, na Europa, e Core entrustable professional activities for entering residency, nos Estados Unidos ${ }^{16}$. As recomendações abrangiam a inclusão de apenas conteúdos considerados essenciais no currículo obrigatório, o ensino de forma integrada e abordagens pedagógicas que associassem a teoria à prática e propiciassem uma aprendizagem significativa, com foco nas competências a serem alcançadas $^{17}$ para o bom desempenho do futuro profissional médico ${ }^{18}$.

No Brasil, um movimento nacional para mudar a educação médica resultou na composição de uma comissão com diversas instituições envolvidas com a educação médica, para avaliação nacional das EM, a Comissão Interinstitucional Nacional de Avaliação do Ensino Médico (Cinaem) ${ }^{19}$. Os achados dessa avaliação embasaram a discussão coletiva sobre as mudanças necessárias na educação médica e resultou, em 2001, na elaboração das Diretrizes Curriculares Nacionais (DCN) para o Curso de Graduação em Medicina ${ }^{20}$, as quais foram revisadas em 2014²1. Entre suas diversas recomendações, as DCN estabelecem que o médico tenha um perfil ético, crítico, humanista e reflexivo, com competência para atuar como médico generalista, atendendo às necessidades de saúde individuais e coletivas da população ${ }^{20,21}$.

Especificamente em relação à cirurgia, as DCN não fornecem conteúdos específicos a seu ensino, mas elencam ações-chave da atenção às necessidades de saúde que a incluem, por exemplo, aventar hipóteses diagnósticas com base no raciocínio clínico-epidemiológico e identificar situações de emergência prevalentes e atuar de forma autônoma e competente nesse contexto . Além disso, as DCN estabelecem que a cirurgia é uma área essencial do internato ${ }^{21}$. Espera-se então que, durante sua formação, o estudante de Medicina desenvolva o raciocínio clínico-cirúrgico, diagnostique patologias eventualmente cirúrgicas e adquira habilidades para realizar procedimentos cirúrgicos básicos, como sutura, drenagens e punções, necessários ao futuro profissional médico generalista ${ }^{7,8}$.

A CH e os conteúdos de cirurgia têm gerado debates e estudos internacionais. No Reino Unido, um consenso sobre o conteúdo de cirurgia para o currículo das EM elaborado por cirurgiões ${ }^{22}$ considerou que o tempo dedicado ao ensino de cirurgia em seus cursos de Medicina era insuficiente ${ }^{22}$, sendo necessário garantir que a formação tornasse os estudantes competentes em procedimentos cirúrgicos básicos ${ }^{23}$. Nos Estados Unidos, outra preocupação relacionada à $\mathrm{CH}$ de cirurgia foi a diminuição de médicos que escolhiam carreiras cirúrgicas, constatada após redução de $30 \%$ na $\mathrm{CH}$ obrigatória de cirurgia durante o internato nas escolas norte-americanas durante a década de $1990^{24,25}$.

No Brasil, as DCN não fornecem limites de $\mathrm{CH}$ antes do internato, nem para cirurgia, nem para outras áreas ${ }^{21}$. Se, por um lado, isso permite flexibilidade às EM no planejamento de seus currículos, por outro, há risco de déficit ou excesso de determinados conteúdos em uma área do saber, na dependência de seus gestores e professores. Como afirma Lima-Gonçalves ${ }^{26}$, a $\mathrm{CH}$ atribuída a cada disciplina é de fundamental importância na estrutura curricular, mas tem sido usada como uma moeda de troca na construção dos currículos, para garantir a representatividade de cada disciplina no curso médico, muitas vezes, sem que todos os seus conteúdos sejam essenciais para a formação.

Em Cuba, as diretrizes nacionais, redigidas pelo Ministério 
da Educação, requerem que as EM cubanas formem médicos competentes para realizar cirurgias das patologias prevalentes na população. Para tanto, as diretrizes estabelecem que a $\mathrm{CH}$ de cirurgia seja de 514,0 horas antes do internato e de 416,0 horas no internato, totalizando uma $\mathrm{CH}$ de 930,0 horas $^{27}$.

Estudo com 26 EM de 16 países da Europa verificou que a CH mínima de cirurgia era de 90,0 horas em Bruxelas (Bélgica) e a máxima de 590,0 horas em Atenas (Grécia)4. Outro estudo com 16 EM de seis países do sudeste europeu encontrou variação de $\mathrm{CH}$ de cirurgia antes do internato, com os limites de 180,0 horas em Osijek (Croácia) e 480,0 horas em Sofia (Bulgária) ${ }^{28}$.

Estudo nos Estados Unidos realizado pela Association of American Medical Colleges (AAMC) incluindo 153 EM constatou a média de 270,0 horas de $\mathrm{CH}$ de cirurgia antes do internato e de 720,0 horas no internato, totalizando a $\mathrm{CH}$ de cirurgia de 990,0 horas $^{29}$.

No Brasil, o curso de Medicina da Universidade Federal de Santa Catarina (UFSC) mudou seu currículo em 2003 para se alinhar às $\mathrm{DCN}^{21,30}$. O currículo é modular integrado, e a cirurgia de adultos tem sido ministrada longitudinalmente no Módulo de Saúde do Adulto, e a cirurgia pediátrica, no Módulo de Saúde da Criança. A CH total do currículo do curso de Medicina é de 7.670,0 horas, sendo a $\mathrm{CH}$ total de cirurgia de 968,0 horas, composta por 355,0 horas antes do internato, incluindo 90,0 horas de Técnica Operatória e Cirurgia Experimental (Toce) e 613,0 horas no internato.

A lacuna existente na literatura sobre a $\mathrm{CH}$ de cirurgia nas EM brasileiras, parte importante do currículo dos cursos de graduação em medicina, levou-nos a questão de pesquisa:

- Como está distribuída a $\mathrm{CH}$ de cirurgia nas $\mathrm{EM}$ brasileiras?

Para responder a essa questão, este estudo foi realizado com o objetivo de analisar a $\mathrm{CH}$ de cirurgia em EM brasileiras.

\section{MÉTODO}

\section{Delineamento e preceitos éticos}

Este estudo foi transversal e descritivo. O projeto de pesquisa não foi submetido ao comitê de ética porque os dados utilizados são de domínio público. Não há conflitos de interesse.

\section{Universo e amostra}

O universo foi constituído por 323 EM brasileiras existentes até setembro de 2018 e reconhecidas pelo Ministério da Educação (MEC), listadas na página do e-MEC ${ }^{31}$, no site http://emec.mec.gov.br. Os critérios de inclusão foram: ter iniciado as atividades acadêmicas antes de 31 de dezembro de 2017 segundo o registro no e-MEC; ter matriz curricular ou projeto político-pedagógico (PPP) disponível na internet; ter informações precisas sobre a $\mathrm{CH}$ de cirurgia.

\section{Coleta de dados}

A coleta dos dados ocorreu de maio a setembro de 2018, com as informações disponíveis na página oficial de cada instituição, a partir da página do e-MEC ${ }^{31}$. Quando não encontrávamos a matriz curricular ou o PPP na página oficial das escolas, fizemos busca na internet com as palavras-chave "nome da universidade", "medicina" e "matriz curricular" ou "projeto político pedagógico ou projeto pedagógico do curso".

As variáveis coletadas foram tipo de currículo, região geográfica e vínculo administrativo da escola, $\mathrm{CH}$ total do curso, $\mathrm{CH}$ de cirurgia antes do internato em disciplinas obrigatórias do curso e $\mathrm{CH}$ de cirurgia no internato médico.

Padronizamos as CHs em "hora/relógio", correspondente a uma hora real de 60 minutos. Quando a escola especificava que a hora/aula era diferente da hora/relógio, fizemos sua conversão, pois, em alguns casos, a hora/aula correspondia a 50 ou 45 minutos reais.

Para definirmos as disciplinas a serem inclusas como cirúrgicas, consideramos a disciplina de Cirurgia Geral e áreas de cirurgia da matriz de competências dos programas de residência médica em cirurgia geral e do programa de pré-requisito em área cirúrgica básica elaboradas pela Comissão Nacional de Residência Médica (CNRM) ${ }^{32}$, que incluíam: Cirurgia do Aparelho Digestivo, Cirurgia Plástica, Cirurgia de Cabeça e Pescoço, Cirurgia Oncológica, Cirurgia Vascular, Cirurgia Torácica, Cirurgia Pediátrica, Cirurgia Cardíaca, Coloproctologia e Urologia. Além dessas, incluímos as disciplinas de Toce e Cirurgia Ambulatorial por serem notadamente disciplinas cirúrgicas.

\section{Análise de dados}

Os dados foram inseridos em banco de dados e analisados com o software SPSS Statistics 26.0 Premium.

A análise dos dados foi realizada por estatística descritiva, com frequência absoluta e relativa para dados categóricos, medidas de tendência central, calculando-se média e desvio padrão (DP) e intervalo de confiança de 95\% (IC95\%) para variáveis de distribuição normal e mediana e percentis 25 e $75\left(P_{25-75}\right)$, quando a distribuição não era normal. Foram também fornecidos os limites mínimos e máximos, quando considerados apropriados. A normalidade da distribuição das variáveis contínuas foi avaliada pelo teste Kolmogorov-Smirnov, considerando-se a distribuição normal quando $p \geq 0,05$. Para comparar escores de variáveis contínuas paramétricas entre dois grupos foi usado o teste $t$ de Student $(t) e$, entre mais de dois grupos a análise de variância (F). Para comparação de escores de variáveis contínuas não paramétricas entre dois 
grupos, foi usado o teste de Mann-Whitney $U(U)$ e, entre mais de dois grupos, o qui-quadrado $\left(\mathrm{X}^{2}\right)$ no teste de Kruskal-Wallis. Os resultados dos testes estatísticos são fornecidos com os graus de liberdade usado, colocados entre parênteses.

Rejeitamos a hipótese nula quando $\mathrm{p}<0,05$.

\section{RESULTADOS}

Do universo de 323 EM, foi possível encontrar a $\mathrm{CH}$ total do curso de 205 que continham alguma carga de cirurgia disponvíel na internet $(63,3 \%)$. Entre as 205,175 escolas $(85,4 \%)$ forneciam a $\mathrm{CH}$ do internato de cirurgia, 157, de cirurgia antes do internato (76,6\%), e 129 forneciam informação a $\mathrm{CH}$ dos dois períodos $(62,9 \%)$.

O currículo das 157 escolas que especificavam sua $\mathrm{CH}$ de cirurgia antes do internato era tradicional em 81 (51,6\%), baseado em problemas (problem based learning - PBL) em 11 (7,0\%), modular em oito $(5,1 \%)$, misto em três $(1,9 \%)$, integrado em uma $(0,6 \%)$, seriado em uma $(0,6 \%)$ e não especificado em $52(33,1 \%)$, e, devido à predominância de currículos tradicionais ou não especificados, essa variável não foi usada para outras análises.

A CH da Toce teve distribuição não normal ( $p=0,000$ em teste de Kolmogorov-Smirnov), com mediana de 90,0 horas $\left(P_{25-}\right.$ $\left.{ }_{75}=60,0-120,0\right)$, média de 96,2 horas (DP $=45,2$; IC 95\% = 87,2105,2 ) e os limites de horas entre 30,0 e 300,0.

O número de EM brasileiras com CH de cirurgia disponível na internet, segundo a região geográfica, administração e gratuidade, é apresentado na Tabela 1.

As $\mathrm{CHs}$ de cirurgia antes do internato, no internato, do total de cirurgia e do total do curso são apresentadas na Tabela 2.

A porcentagem das $\mathrm{CH}$ s antes do internato e durante o internato em relação à $\mathrm{CH}$ total do curso é apresentada na Tabela 3.

A Tabela 4 exibe as $\mathrm{CHs}$ de cirurgia antes do internato, do internato e seu total entre as 129 EM que continham todas essas variáveis disponíveis na internet, por região geográfica e gratuidade do curso. Como pode ser observado, não houve diferença estatística nas $\mathrm{CHs}$ por região geográfica. Também não houve diferença nas $\mathrm{CH}$ s total de cirurgia, nem de cirurgia antes do internato por gratuidade. A única diferença encontrada foi a maior $\mathrm{CH}$ mediana do internato em escolas gratuitas.

\section{DISCUSSÃO}

No presente estudo, constatamos uma variação entre os valores mínimos e máximos da $\mathrm{CH}$ de cirurgia antes do internato e durante esse período nas escolas brasileiras estudadas. Entretanto, a média da $\mathrm{CH}$ antes do internato e a mediana da $\mathrm{CH}$ total de cirurgia foram semelhantes por região geográfica e gratuidade da escola. A única diferença que encontramos foi a maior mediana da $\mathrm{CH}$ de cirurgia do internato nas escolas gratuitas.
Tabela 1. Número de escolas médicas brasileiras com carga horária de cirurgia disponível na internet, segundo a região geográfica, administração e gratuidade

\begin{tabular}{cccc}
\hline & $\begin{array}{c}\text { Escolas } \\
\text { com carga } \\
\text { horária de } \\
\text { cirurgia } \\
\text { antes do } \\
\text { internato }\end{array}$ & $\begin{array}{c}\text { Escolas } \\
\text { com carga } \\
\text { horária de } \\
\text { cirurgia } \\
\text { durante o } \\
\text { internato }\end{array}$ & $\begin{array}{c}\text { Escolas } \\
\text { com carga } \\
\text { horária de } \\
\text { cirurgia em } \\
\text { ambos os } \\
\text { períodos }\end{array}$ \\
Características & $\mathbf{n ( \% )}$ & $\mathbf{n}(\%)^{\mathbf{a}}$ & $\mathbf{n}(\%)$ \\
\hline Região & $16(10,2)$ & $20(11,4)$ & $13(10,1)$ \\
Norte & $28(17,8)$ & $42(24,0)$ & $22(17,0)$ \\
Nordeste & $10(6,4)$ & $14(8,0)$ & $9(7,0)$ \\
Centro-Oeste & $72(45,9)$ & $72(41,1)$ & $59(45,7)$ \\
Sudeste & $31(19,7)$ & $27(15,4)$ & $26(20,2)$ \\
Sul & & & \\
\hline Vínculo & $48(30,6)$ & $68(38,9)$ & $43(33,3)$ \\
Federal & $16(10,2)$ & $21(12,0)$ & $13(10,1)$ \\
Estadual & $5(3,2)$ & $6(3,4)$ & $4(3,1)$ \\
Municipal & $88(56,0)$ & $80(45,7)$ & $69(53,5)$ \\
Privada & & & \\
\hline Gratuidade & $64(40,8)$ & $89(50,9)$ & $56(43,4)$ \\
Sim & $157(100,0)$ & $175(100,0)$ & $129(100,0)$ \\
\hline Não & $93(59,2)$ & $86(49,1)$ & $73(56,6)$ \\
\hline Total & & &
\end{tabular}

Abreviaturas - $\mathrm{n}$ (\%): número (frequência absoluta) e porcentagem (frequência relativa).

${ }^{\text {aA } A}$ porcentagem total varia de $99,9 \%$ a $100 \%$ em razão do arredondamento para uma casa decimal.

Fonte: Elaborada pelos autores.

Os limites extremos encontrados de $\mathrm{CH}$ de cirurgia nos fizeram refletir quanto à possibilidade de que, enquanto, em algumas escolas, o conteúdo teórico e/ou prático de cirurgia poder estar muito baixo, o que poderia resultar em um preparo insuficiente do estudante para alcançar as competências requeridas nessa área, em outras, esse conteúdo pode estar excessivo, não sendo essencial para a formação, podendo representar uma causa a mais de sobrecarga para os estudantes.

Quanto à maior mediana na $\mathrm{CH}$ do internato em escolas gratuitas, é difícil explicarmos tal achado porque a definição dessa $\mathrm{CH}$ pode ser influenciada por vários fatores. Como não houve diferença estatística na $\mathrm{CH}$ total de cirurgia por gratuidade, uma possível razão poderia ser a forma como o conteúdo teórico-prático é distribuído no currículo dessas escolas. Entretanto, explicações mais consistentes só poderiam ser dadas após investigação de vários outros aspectos além da carga horária do curso. Alguns desses, por exemplo, seriam a visão da escola sobre o valor da área cirúrgica (que poderia ser avaliada no PPP e por entrevistas com os gestores), o período de 
Tabela 2. Cargas horárias de cirurgia e de todo o currículo nas escolas médicas brasileiras que as disponibilizavam na internet até setembro de 2018

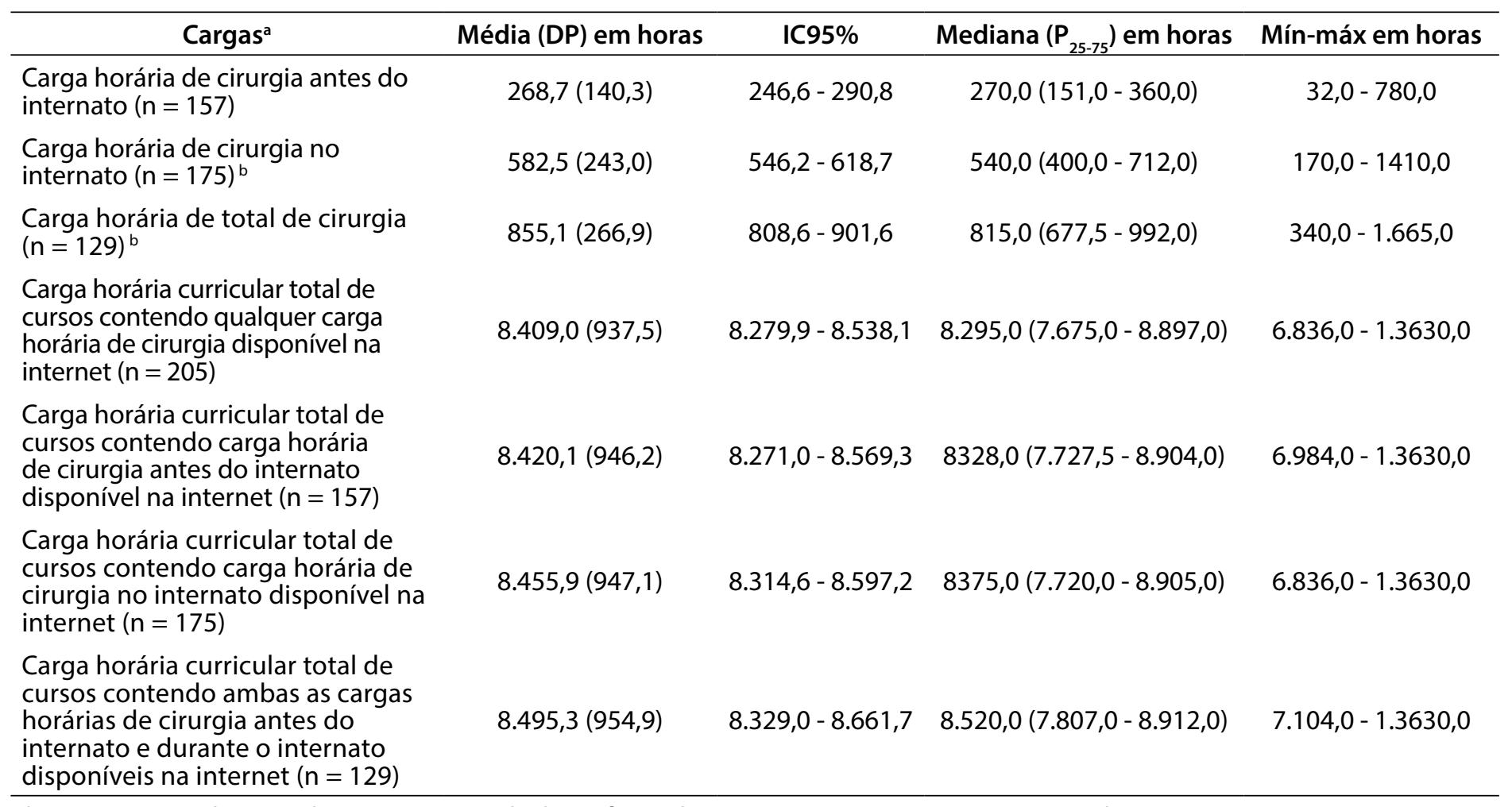

Abreviaturas - DP: desvio padrão; IC95\%: intervalo de confiança de 95\%; $\mathrm{P}_{25-75}$ : percentis 25 e 75; mín-máx: limites mínimo e máximo.

${ }^{a} \mathrm{~A}$ carga horária está apresentada em horas relógio (hora de 60 minutos) e é fornecida tanto sua média quanto sua mediana para possibilitar comparações com outros estudos.

${ }^{b}$ Distribuição não normal ( $p<0,05$ em teste Kolmogorov-Smirnov), devendo ser considerados a mediana e os percentis 25 e 75 .

Fonte: Elaborada pelos autores.

Tabela 3. Porcentagem da carga horária parcial e total de cirurgia em relação à carga horária total do curso nas escolas médicas que disponibilizavam tanto suas cargas horárias antes quanto durante o internato na internet até setembro de 2018

\begin{tabular}{|c|c|c|c|c|}
\hline Porcentagens & Média (DP) em horas & IC95\% & Mediana $\left(P_{25-75}\right)$ em horas & Mín-máx em horas \\
\hline $\begin{array}{l}\text { Carga horária de cirurgia no } \\
\text { internato em relação à carga horária } \\
\text { curricular total dos cursos com carga } \\
\text { horária de cirurgia no internato } \\
\text { disponível na internet }(n=175)^{\mathrm{a}}\end{array}$ & $6,9(2,7)$ & $6,5-7,3$ & $6,4(5,0-8,2)$ & $1,8-15,1$ \\
\hline $\begin{array}{l}\text { Carga horária de cirurgia antes } \\
\text { do internato somada à durante } \\
\text { o internato em relação à carga } \\
\text { horária curricular total dos cursos } \\
\text { que continham ambas as cargas } \\
\text { disponíveis na internet }(\mathrm{n}=129)^{\mathrm{a}}\end{array}$ & $10,1(3,0)$ & $9,6-10,6$ & $9,7(8,3-11,8)$ & $3,5-18,9$ \\
\hline
\end{tabular}

Abreviaturas - DP: desvio padrão; intervalo de confiança de 95\%; $P_{25-75}$ : percentis 25 e 75; mín-máx: limites mínimo e máximo.

a Distribuição não normal ( $p<0,05$ em teste Kolmogorov-Smirnov), devendo ser considerada a mediana e os percentis 25 e 75 .

Fonte: Elaborada pelos autores. 
Tabela 4. Cargas horárias de cirurgia entre as 129 escolas médicas incluídas no estudo que continham essas cargas disponíveis na internet, segundo a região geográfica e gratuidade da escola

\begin{tabular}{|c|c|c|c|c|c|c|}
\hline Características & $\begin{array}{l}\text { Carga horária } \\
\text { de cirurgia } \\
\text { antes do } \\
\text { internato } \\
\text { Média (DP) } \\
\text { em horas }\end{array}$ & $\begin{array}{l}\text { Resultados } \\
\text { de testes } \\
\text { estatísticos: } \\
\text { teste(gl); } p\end{array}$ & $\begin{array}{c}\text { Carga Horária } \\
\text { de cirurgia no } \\
\text { internato } \\
\text { Mediana }\left(P_{25-75}\right) \text { em } \\
\text { horas }\end{array}$ & $\begin{array}{l}\text { Resultados } \\
\text { de testes } \\
\text { estatísticos: } \\
\text { teste(gl); p }\end{array}$ & $\begin{array}{c}\text { Carga horária total } \\
\text { de cirurgia } \\
\text { Mediana }\left(P_{25-75}\right) \text { em } \\
\text { horas }\end{array}$ & $\begin{array}{l}\text { Resultados } \\
\text { de testes } \\
\text { estatísticos: } \\
\text { teste(gl); } p\end{array}$ \\
\hline
\end{tabular}

\section{Regiãoc}

\begin{tabular}{ccccccc} 
Norte $(\mathrm{n}=13)$ & $275,7(100,2)$ & & $440,0(395,0-742,0)$ & & $784,0(640,0-1.010,0)$ & \\
Nordeste $(\mathrm{n}=22)$ & $235,3(107,2)$ & & $583,5(385,0-714,2)$ & $X^{2}(4)=1,4 ;$ & $812,5(651,2-945,5)$ & $X^{2}(4)=1,4 ;$ \\
Centro-Oeste $(\mathrm{n}=9)$ & $270,6(164,2)$ & $\mathrm{F}(4)=0,4 ;$ & $560,0(492,0-612,5)$ & $\mathrm{p}=0,85$ & $799,0(682,0-983,5)$ & $\mathrm{p}=0,85$ \\
Sudeste $(\mathrm{n}=59)$ & $270,3(153,2)$ & & $570,0(420,0-768,0)$ & & $852,0(705,0-1.080,0)$ & \\
Sul $(\mathrm{n}=26)$ & $286,6(131,9)$ & & $505,0(429,5-664,0)$ & $813,0(675,0-1.008,7)$ & \\
\hline
\end{tabular}

Gratuidade

$\begin{array}{ccccccc}\operatorname{Sim}(n=56) & 249,8(126,2) & t(126)=-1,5 ; & 585,0(427,2-843,0) & U=1.609,0 ; & 865,0(706,5-1.125,2) & U=1.752,5 ; \\ \text { Não }(n=73) & 285,2(140,8) & p=0,14 & 510,0(400,0-647,0) & p=0,04 & 800,0(658,0-915,0) & p=0,17\end{array}$

Abreviaturas - DP: desvio padrão; gl: graus de liberdade; $p$ : valor de significância estatística; $P_{25}$ : percentis 25 e 75 ; F: variância no fator em teste de ANOVA; $\mathrm{X}^{2}$ : qui-quadrado em teste de Kruskal-Wallis; t: teste t de Student; U: teste de Mann-Whitney U.

Fonte: Elaborada pelos autores.

início das práticas em ambulatório e de procedimentos cirúrgicos, os cenários de prática e como os estudantes são alocados nesses cenários, o número de professores/médicos que atuam como preceptores no internato que têm domínio em cirurgia.

Quanto aos valores da $\mathrm{CH}$ de cirurgia, não encontramos estudos nacionais que propiciassem comparação com os achados de nosso estudo. Há documentos nacionais sobre os conteúdos da matriz curricular para fins de revalidação de diplomas de médicos obtidos no exterior ${ }^{33}$, mas sem recomendações de carga horária.. Essa lacuna, na verdade, foi o que nos motivou a desenvolver esta pesquisa, para que os conhecimentos construídos pudessem propiciar futuras comparações, contribuindo assim para o processo de construção e avaliação dos currículos.

Por sua vez, os estudos internacionais propiciam comparação ${ }^{4,27-29}$. Em Cuba, a CH de cirurgia preconizada pelo Ministério da Educação de Cuba antes do internato está acima da média e do IC $95 \%$ encontrados em nosso estudo ea $\mathrm{CH}$ de cirurgia no internato e a total estão dentro dos limites dos percentis de nosso estudo ${ }^{27}$. Isso nos faz refletir sobre a possibilidade de haver um excesso de conteúdos relativos à cirurgia nos currículos das EM brasileiras, pois os médicos cubanos recebem formação para realizar procedimentos cirúrgicos mais amplos, o que no Brasil é praticado apenas por cirurgiões.

No estudo com 16 escolas do sudeste europeu, cinco instituições tiveram uma $\mathrm{CH}$ de cirurgia antes internato abaixo do IC 95\% de nosso estudo, nove acima e duas dentro dos limites de nosso em estudo, uma em Mostar (Bósnia e Herzegovina) e outra em Pristina (Kosovo) ${ }^{28}$.
No outro estudo europeu, com 26 escolas, sete tinham $\mathrm{CH}$ de cirurgia antes do internato abaixo do limite do IC $95 \%$ de nosso estudo, 13 acima e seis dentro do limite de nosso estudo (Split na Croácia; Brno na República Tcheca; Roma, Florença e Nápoles na Itália; e Viena na Áustria) ${ }^{4}$.

Quanto à $\mathrm{CH}$ de cirurgia nas 153 escolas norteamericanas, a média da $\mathrm{CH}$ de cirurgia antes do internato encontra-se dentro do limite do IC $95 \%$ de nosso estudo, a do internato acima do limite do $P_{25-75}$ de nosso estudo e a total de cirurgia dentro do limite do $P_{25-75}$ de nosso estudo ${ }^{29}$.

Percebemos então que há uma grande variação nos países europeus em todas essas cargas e que os limites de percentis 25 e $75 \mathrm{da} \mathrm{CH}$ total de cirurgia nas escolas brasileiras incluem os valores encontrados nas escolas cubanas e norteamericanas. Por sua vez, a CH antes do internato está dentro dos limites da cubanas e abaixo das norte-americanas, enquanto a do internato está dentro dos limites das escolas cubanas e menor do que as norte-americanas.

As limitações de nosso estudo incluíram a impossibilidade de encontrar a $\mathrm{CH}$ de cirurgia em currículos integrados que não a especificavam e de localizar a documentação completa das $\mathrm{CHs}$ das disciplinas. Apesar de se almejar a integração curricular das diversas áreas do conhecimento, idealmente a $\mathrm{CH}$ destinada aos conteúdos poderia ser especificada para fornecer parâmetros objetivos de comparação. Outra limitação de nosso estudo foi a coleta de dados disponíveis na internet, enquanto o ideal seria coletar esses dados diretamente das EM para melhor compreensão sobre a distribuição das $\mathrm{CH}$ s teórica e prática e sobre o conteúdo abordado. 
Finalmente, sugerimos novos estudos para avaliar a corrente de pensamento predominante que guia os currículos das escolas, a percepção de gestores e professores sobre o que é essencial e necessário ensinar na área cirúrgica durante a graduação e a abordagem pedagógica do ensino de cirurgia, incluindo métodos de avaliação discente, cenários de prática e conteúdos entre outros aspectos.

Nosso estudo sobre a CH propicia a identificação do valor/ peso dado à cirurgia no currículo das escolas estudadas e contribui para que gestores e responsáveis pela educação médica tenham parâmetros sobre como está a $\mathrm{CH}$ de cirurgia nas escolas brasileiras. Entretanto, estes achados devem ser considerados como a "ponta do iceberg", não sendo suficientes para a análise da qualidade destes cursos de medicina e dos fatores que influenciam a decisão sobre a CH da cirurgia em cada escola ${ }^{34,35}$.

\section{CONCLUSÕES}

Respondendo aos objetivos de nosso estudo, apesar de os limites extremos da $\mathrm{CH}$ de cirurgia serem bastante variáveis, não há diferença estatística nem na $\mathrm{CH}$ média de cirurgia antes do internato, nem na mediana da $\mathrm{CH}$ total de cirurgia por região geográfica e gratuidade das escolas, e não há diferença estatística na mediana da $\mathrm{CH}$ de cirurgia no internato por região geográfica. Entretanto, a maior $\mathrm{CH}$ de cirurgia no internato é significantemente maior nas escolas gratuitas.

Os dados de nosso estudo podem ajudar os responsáveis pela educação médica a compreender a distribuição da $\mathrm{CH}$ de cirurgia nas EM brasileiras e auxiliar os gestores de EM na avaliação e no planejamento da $\mathrm{CH}$ de cirurgia em suas escolas.

Sugerimos que futuros estudos incluam mais informações, desde a concepção político-pedagógica do curso até os cenários e métodos de ensino e avaliação do ensino de cirurgia, com dados coletados tanto em documentos das escolas como por meio de entrevistas ou grupos focais com os envolvidos no seu processo de ensino-aprendizagem.

\section{CONTRIBUIÇÃO DOS AUTORES}

Athos Paulo Santos Martini participou na elaboração do projeto, na coleta e análise de dados, ena redação e revisão do manuscrito, que foi seu trabalho de conclusão de curso, apresentado como requisito do curso de graduação em Medicina da UFSC. Sofia Romay Oliveira participou na elaboração do projeto, na coleta e análise de dados, e na redação do manuscrito. Suely Grosseman participou na orientação de Athos Paulo Santos Martini, na elaboração do projeto, na coleta e análise de dados, na confecção do projeto e na redação e revisão do manuscrito.

\section{CONFLITO DE INTERESSES}

Declaramos não haver conflito de interesses neste estudo.

\section{FINANCIAMENTO}

Declaramos que não houve financiamento neste estudo.

\section{REFERENCES}

1. Agha RA, Papanikitas A, Baum M, Benjamin IS. The teaching of surgery in the undergraduate curriculum-reforms and results. Int J Surg 2005;3(1):87-92.

2. Agha RA, Papanikitas A, Baum M, Benjamin IS. Part II-importance and recommendations for change. Int. J Surg. 2005, 3(2), 151-157.

3. Baum M. Teaching the humanities to medical students. Clin Med. 2002;2(3):246-249.

4. Dušek T, Bates T. Analysis of European medical schools' teaching programs Croat Med J. 2003;44:26-31.

5. Bohoslavsky R. Lo vocacional: teoría, técnica e ideología. Buenos Aires: Ediciones Búsqueda; 1975.

6. Flexner A. Medical education in the United States and Canada: a report to the Carnegie Foundation for the advancement of teaching. Bulletin number four. New York: The Carnegie Foundation for the Advancement of Teaching; 1910 [access in 20 aug 2020]. Available from: http://archive. carnegiefoundation.org/publications/pdfs/elibrary/Carnegie_Flexner_ Report.pdf.

7. DiCarlo SE. Too much content, not enough thinking, and too little FUN! Adv Physiol Educ. 2009;33(4):257-64.

8. Andersen S, Stentoft D, Emmersen J, Rasmussen S, Birkelund S, Nøhr S (2019). Contention over undergraduate medical curriculum content. Int J Med Educ. 2019;10: 230-231.

9. Jonas HS, Etzel SI, Barzansky B. Undergraduate medical education. JAMA. 1990;264(7):801-9.

10. General Medical Council. Tomorrow's doctors: recommendations on undergraduate medical education. London: GMC; 1993 [access in 20 aug 2020]. Available from: https://www.yumpu.com/es/document/ $\mathrm{read} / 47554869 /$ tomorrows-doctors-1993-general-medical-council.

11. General Medical Council. Tomorrow's doctors: outcomes and standards for undergraduate medical education. London: GMC; 2009 [access in 18 jun 2020]. Available from: http:// www.ub.edu/medicina unitateducaciomedica/documentos/TomorrowsDoctors_2009.pdf.

12. Frank JR, Danoff D. The CanMEDS initiative: implementing an outcomesbased framework of physician competencies. Med Teach. 2007;29(7):642-7.

13. Frank JR, Snell L, Sherbino J. Draft CanMEDS 2015: physician competency framework - Series IV. Ottawa: The Royal College of Physicians and Surgeons of Canada; 2015 [access in 18 jun 2020]. Available from: http:// www.royalcollege.ca/portal/page/portal/rc/common/documents/ canmeds/framework/ canmeds2015_framework_series_IV_e.pdf.

14. Harden RM. AMEE Guide no 14: outcome-based education: Part 1-An introduction to outcome-based education. Med Teac. 1999;21(1):7-14.

15. World Federation for Medical Education in Europe, University of Copenhagen, Association for Medical Education in Europe. Statement on the Bologna Process and Medical Education. 2005 [access in 18 jun 2020]. Available from: http://www.aic.lv/bolona/Bologna/contrib/Statem_oth/ WFME-AMEE.pdf.

16. Obeso V, Brown D, Aiyer M. Core entrustable professional activities for entering residency: toolkits for the 13 core EPAs. Washington, DC: Association of American Medical Colleges; 2017 [access in 10 set 2020]. Available from: https://www.aamc.org/what-we-do/mission-areas/ medical-education/cbme/core-epas/publications.

17. Gomes AP, Rego S. Transformação da educação médica: é possível formar um novo médico a partir de mudanças no método de ensinoaprendizagem? Rev Bras Educ Med. 2011;35(4):557-66.

18. Bollela VR, Machado JLM. O currículo por competências e sua relação com as diretrizes curriculares nacionais para a graduação em medicina. Science in Health. 2010;1(2):126-42. 
19. Comissão Interinstitucional Nacional de Avaliação do Ensino Médico. Preparando a transformação da educação médica brasileira: projeto CINAEM III fase relatório 1999-2000. Piccini RX, Facchini LA, Carvalho dos Santos R, organizadores..Pelotas: UFPel; 2000.

20. Brasil. Resolução CNE/CES n 4, de 7 de novembro de 2001. Institui Diretrizes Curriculares Nacionais do Curso de Graduação em Medicina. Diário Oficial da União, Brasília; 9 nov 2001. Seção 1, p. 38.

21. Brasil. Resolução CNE/CES n 3, de 20 de junho de 2014. Institui Diretrizes Curriculares Nacionais do Curso de Graduação em Medicina e dá outras providências. Diário Oficial da União, Brasília; 23 jun 2014. Seção 1, p. 8-11.

22. Baker D, Deekonda P, Glasbey J, Humm G, Mohan H, Nally D, et al. ASiT Medical Student and Foundation Doctor Consensus Group. Core content of the medical school surgical curriculum: consensus report from the Association of Surgeons in Training (ASIT). Int J Surg. 2020, 84:186-193.

23. Hakim MA, Dominguez ED, Priest $S$, Lee KS, Mardanpour A, Tandle S, et al. Surgical skills workshops should be a part of the United Kingdom undergraduate medical curriculum. Cureus. 2019;11(5): e4642.

24. Polk HC Jr. The declining interest in surgical careers, the primary care mirage, and concerns about contemporary undergraduate surgical education. Am J Surg. 1999;178(3):177-9.

25. Peel JK, Schlachta CM, Alkhamesi NA. A systematic review of the factors affecting choice of surgery as a career. Can J Surg. 2018;61(1):58-67.

26. Lima-Gonçalves L. Médicos e ensino da medicina no Brasil. São Paulo: Edusp; 2002.

27. Miralles AEÁ, Taureaux DN, Fernández SJA, Pernas GM, Sierra FS, Diego CJM. Cronología de los mapas curriculares en la carrera de Medicina. Rev Cuba Educ Méd Super. 2015;29(1):93-107.

28. Likic R, Dusek T, Horvat D. Analysis and prospects for curricular reform of medical schools in Southeast Europe. Med Educ. 2005;39(8):833-40.
29. Association of American Medical Colleges. Curriculum Reports. AAMC Curriculum Inventory, 2018-2019. Washington, DC: AAMC; 2020 [access in 17 aug 2020]. Available from: https://www.aamc.org/data-reports/ curriculum-reports/report/curriculum-reports.

30. Pereima MJL, Coelho EBS, Da Ros MA. Da proposta à ação: currículo integrado do curso de medicina da UFSC. Florianópolis: Editora da UFSC; 2005.

31. Ministério da Educação. Cadastro Nacional de Cursos e Instituições de Educação Superior: cadastro e-MEC. Brasília; 2018 [access in 18 jun 2020]. Available from: http://emec.mec.gov.br/.

32. Brasil. Resolução no 48 , de 28 de junho de 2018. Dispõe sobre a Matriz de Competências dos Programas de Residência Médica em Cirurgia Geral e do Programa de Pré-requisito em Área Cirúrgica Básica no Brasil. Brasília: Comissão Nacional de Residência Médica, Secretaria de Educação Superior; 2018.

33. Brasil. Matriz de correspondência curricular para fins de revalidação de diplomas de médico obtidos no exterior. Brasília: Ministério da Educação, Ministério da Saúde; 2009 [access in 18 jun 2020]. Available from: http:// download.inep.gov.br/educacao_superior/revalida/matriz/2009/matriz correspondencia_curricular_revalida_sem_logo.pdf.

34. Del Ciampo LA, Del Ciampo IRL. Curso de medicina e ensino de pediatria nas escolas médicas brasileiras. Pediatria (São Paulo). 2010;32:9-14.

35. Cutolo LRA, Delizoicov D. O currículo do curso de graduação em medicina da UFSC: análise a partir das categorias Fleckianas. II Encontro Nacional de Pesquisa em Educação em Ciências; 1999; Valinhos. Valinhos: Associação Brasileira de Pesquisa em Educação; 1999. 14 p. [aceso em 18 jun 2020]. Available from: http://abrapecnet.org.br/atas_enpec/iienpec/Dados/ trabalhos/G31.pdf. 